\title{
Measurement of vertebral endplate bone marrow lesion (Modic change) composition with water-fat MRI and relationship to patient-reported outcome measures
}

\author{
Aaron J. Fields ${ }^{1} @$ - Alexander Ballatori ${ }^{1} \cdot$ Misung Han $^{2}$. Jeannie F. Bailey ${ }^{1} \cdot$ Zachary L. McCormick $^{3}$. \\ Conor W. O'Neill ${ }^{1} \cdot$ Sibel Demir-Deviren ${ }^{1} \cdot$ Roland Krug $^{2} \cdot$ Jeffrey C. Lotz ${ }^{1}$
}

Received: 26 October 2020 / Revised: 22 December 2020 / Accepted: 17 January 2021 / Published online: 6 February 2021

(C) The Author(s) 2021

\begin{abstract}
Purpose Vertebral endplate bone marrow lesions ("Modic changes", MC) are associated with chronic low back pain (CLBP). Bone marrow composition in MC is poorly understood. The goals of this study were to: (1) measure bone marrow fat fraction (BMF) in CLBP patients with MC using water-fat MRI and (2) assess the relationship between BMF measurements and patient-reported clinical characteristics.

Methods In this cross-sectional study, 42 CLBP patients (men, $n=21$; age, $48 \pm 12.4$ years) and 18 asymptomatic controls (men, $n=10 ; 42.7 \pm 12.8$ years) underwent $3 \mathrm{~T}$ MRI between January 2016 and July 2018. Imaging consisted of $\mathrm{T}_{1^{-}}$and $\mathrm{T}_{2}$-weighted sequences to evaluate $\mathrm{MC}$ and spoiled gradient-recalled echo sequence with asymmetric echoes and leastsquares fitting to measure BMF. BMF was compared between vertebrae with and without MC using mixed effects models. The relationship between the BMF measurements and patient-reported disability scores was examined using regression.

Results Twenty-seven subjects (26 CLBP, 1 control) had MC, and MC presence coincided with significantly altered BMF. In MC 1, BMF was lower than endplates without MC (absolute difference $-22.3 \% ; p<0.001$ ); in MC 2, BMF was higher (absolute difference 21.0\%; $p<0.001$ ). Absolute BMF differences between affected and unaffected marrow were larger in patients with greater disability $(p=0.029-0.032)$ and were not associated with pain $(p=0.49-0.83)$.

Conclusion BMF is significantly altered in MC. Water-fat MRI enables BMF measurements that may eventually form the basis for quantitative assessments of MC severity and progression.
\end{abstract}

Keywords Bone marrow $\cdot$ Chronic low back pain $\cdot$ Magnetic resonance imaging phenotypes $\cdot$ Endplate $\cdot$ Modic changes

\section{Introduction}

Assessment of vertebral endplate bone marrow lesions (BML) is an important clinical task because of the positive association between MRI evidence of endplate BML and chronic low back pain (CLBP) [1, 2]. For example, BML

Aaron J. Fields

aaron.fields@ucsf.edu

1 Department of Orthopaedic Surgery, University of California, 513 Parnassus Avenue, San Francisco, CA 116194143-0514, USA

2 Department of Radiology and Biomedical Imaging, University of California, San Francisco, CA, USA

3 Department of Physical Medicine and Rehabilitation, University of Utah School of Medicine, Salt Lake City, UT, USA characteristics such as size, location, and type, i.e. fibrovascular versus fatty, may provide information for diagnosis, prognosis, and treatment planning [3-5]. The most widely used methodology for assessing endplate BML is based on the presence of signal intensity changes adjacent to the endplates on $T_{1}$ - and $T_{2}$-weighted MRI, often called "Modic changes" (MC) [6]. A limitation of these anatomical sequences is that they provide only qualitative visual information about lesion structure, and they are not sensitive to subtle variations in marrow composition. Thus, marrow composition in endplate BML remains poorly understood. For example, it is unknown how BML composition varies within or between patients, relates to clinical symptoms, or changes during BML progression. Such knowledge could form the basis for objective and continuous measures of endplate BML, inform new treatments, and provide targets for monitoring efficacy. 
One unexplored approach for evaluating marrow composition in endplate BML is based on chemical shift encodingbased water-fat imaging. This technique enables spatially resolved assessments of bone marrow fat fraction (BMF) [7]. In water-fat imaging, water and fat signals can be separated based on the chemical shift difference between water and fat, and accurate fat quantification can be achieved by incorporating a multi-peak fat spectrum in the signal model [8]. Prior studies using water-fat imaging reported vertebral BMF values that demonstrate good agreement with histological results $[9,10]$ and showed age-related increases in BMF that are consistent with natural conversion of red to yellow marrow [11]. The purposes of this study were to measure BMF in CLBP patients with endplate BML using water-fat MRI and to assess the relationship between BMF measurements and patient-reported clinical characteristics.

\section{Material and method}

This prospective cross-sectional study was approved by the site's institutional review board (\#13-12224; approved August 21, 2014). Informed consent was obtained from each subject.

\section{Subjects}

Between January 2016 and July 2018, we enrolled forty-two patients with CLBP (more than three consecutive months with low back pain; VAS $\geq 4$ or ODI $\geq 30$ ) and eighteen control subjects without prior history of back pain or spinal pathology (VAS $\leq 1$ ). Major exclusion criteria were pregnancy, diabetes, positive smoking status, cancer, spondylolisthesis, scoliosis, prior lumbar surgery, disc herniation, compression fracture, and active use of osteoporosis medication(s). A subset of the subjects was included in previous studies that did not evaluate MC (forty-six subjects [12]) or vertebral BMF (fifty-two subjects [13]). Demographic information is summarized in Table 1.

\section{Imaging}

Lumbar MRI was performed on a Discovery MR 750 3-T scanner using an 8-channel phased-array spine coil (GE Healthcare). Clinical fast spin echo images with $T_{1}$ and $\mathrm{T}_{2}$ weighting were acquired in the sagittal orientation (see Detailed Methods, Supplemental Material).

Water-fat imaging consisted of a 3D spoiled gradientrecalled echo (SPGR) sequence with six echoes and iterative decomposition of water and fat with echo asymmetry and least-squares estimation (IDEAL) reconstruction algorithm [7, 14] (see Detailed Methods, Supplemental Material). To test for repeatability of water-fat imaging and analysis, two
Table 1 Demographics of chronic low back pain patients and asymptomatic control subjects

\begin{tabular}{lllr}
\hline Characteristic & $\begin{array}{l}\text { Control subjects } \\
(n=18)\end{array}$ & $\begin{array}{l}\text { Back pain } \\
\text { patients } \\
(n=42)\end{array}$ & $P$ value \\
\hline Age (year) & $42.7 \pm 12.8$ & $48.0 \pm 12.4$ & 0.15 \\
Male & $10(55.5)$ & $21(50.0)$ & 0.69 \\
Weight $(\mathrm{kg})$ & $71.3 \pm 12.7$ & $76.6 \pm 17.6$ & 0.21 \\
Height $(\mathrm{cm})$ & $173.7 \pm 10.0$ & $173.3 \pm 9.9$ & 0.89 \\
BMI $\left(\mathrm{kg} / \mathrm{m}^{2}\right)$ & $23.6 \pm 4.2$ & $25.5 \pm 4.9$ & 0.17 \\
Clinical measures & & & \\
ODI & $0 \pm 0$ & $33.3 \pm 14.4$ & $<0.001$ \\
VAS & $0 \pm 0$ & $6.6 \pm 1.7$ & $<0.001$ \\
Pfirrmann grade & $2.2 \pm 1.0$ & $3.0 \pm 1.1$ & $<0.001$ \\
MC 1 & $0(0)$ & $26(5.9)$ & $<0.001$ \\
MC 2 & $2(1.3)$ & $47(11.2)$ & $<0.001$ \\
MC 3 & $0(0)$ & $0(0)$ & 0.99 \\
\hline
\end{tabular}

For all characteristics except male sex and number of levels with each type of MC, data are shown as mean \pm SD. Data in parentheses are percentages. Independent $t$ tests were used to compare the differences between two groups for continuous numerical variables. $\chi^{2}$ tests were used to compare the differences between two groups for sex and for the clinical measures. BMI body mass index, ODI Oswestry Disability Index score; VAS Visual Analogue Scale score, MC Modic changes

subjects were each scanned three times on the same day, having exited and re-entered the scanner between scans.

\section{Image analysis}

BMF maps were generated from the six acquired echoes using the validated IDEAL algorithm that accounts for multiple peaks in the fat spectrum and performs $\mathrm{T}_{2} *$ correction $[14,15]$. The maps were analysed two ways. In the first analysis, BMF values were averaged for five circular ROIs $\left(10.08 \mathrm{~mm}^{2}\right.$ area) randomly placed inside the trabecular bone encompassed by the endplate BML, using conventional MC grading (see below) as a guide for ROI placement on the BMF maps. The BMF measurements inside the endplate BML were compared to BMF measurements averaged for five circular ROIs placed outside the BML in the adjacent, unaffected trabecular bone. For levels without endplate BML, the BMF values were averaged for five mid-sagittal ROIs placed in the trabecular bone adjacent to the endplate. The ROI analysis was performed in the 10 lumbosacral endplates from the inferior L1 endplate to the superior S1 endplate in each subject (600 endplates total).

In the second analysis, the five lumbar vertebral bodies plus first sacral vertebral body were manually segmented on the five mid-sagittal slices in IDL 8.7 (Harris Geospatial Solutions) to determine the distribution of vertebral BMF values at each level. Parameters derived from the BMF 
distributions-mean, median, standard deviation, coefficient of variation (SD/mean), skewness, and full width at half maximum-were compared between levels with and without endplate BML in order to understand how BML presence impacts the overall BMF distribution within the vertebral body. The whole-vertebral analysis was performed for six lumbosacral vertebrae in each subject (L1-S1; 360 vertebrae total).

\section{Clinical grading}

Assessment of endplate BML and disc Pfirrmann grade was performed by two raters blinded to group. Both are boardcertified subspecialists in Pain Medicine with 20 years (C.O.) and 8 years (Z.M.) of experience reading and interpreting spine MRIs for clinical and research purposes. Endplate BML grading was done on $\mathrm{T}_{1}$-and $\mathrm{T}_{2}$-weighted images according to standard MC definitions [2], and all $\mathrm{MC}$ cases were large enough for ROI placement. To gauge inter-rater reliability, both raters scored a subset of the endplates (90/600). Subjects completed VAS and ODI to assess clinical symptoms.

\section{Statistical analysis}

Statistical analysis was performed using JMP Pro 14 (SAS Institute). A two-sided $p$ value less than 0.05 was considered significant. The Kappa statistic was used to assess inter-rater reliability of MC classification. Independent $t$ tests and $\chi^{2}$ tests were used to compare differences between two groups for continuous and dichotomous variables, respectively. Mixed effects models accounting for multiple measurements per subject were used to estimate the least squares mean of the various BMF parameters [16], and Tukey's HSD post hoc tests were used to test for differences in the least squares mean between groups. Paired $t$ tests were used to test for BMF differences between affected and unaffected regions of the same vertebral body. Parametric tests were used since the data did not violate the normality assumption ( $p>0.05$, Anderson-Darling test).

To test associations between BMF measures (endplatelevel variables) and clinical outcomes (subject-level variables), we derived two composite BMF measures for each patient with MC, using the absolute differences in $\mathrm{BMF}$ values between affected and unaffected marrow from the ROI analysis. The first BMF measure was the mean difference in BMF between affected and unaffected marrow for all MC-affected endplates in a particular patient; the second measure was the maximum difference. Linear regression and logistic regression were used to assess relationships between the BMF measures and ODI and VAS, respectively.

Repeatability of BMF mapping was characterized by the precision error, which was defined as the root-mean-square average of the precision errors in mean BMF for subjects who underwent repeat MRI [17].

\section{Results}

\section{Study population characteristics}

No significant differences were found in age, sex, weight, height, or BMI between CLBP patients and controls (Table 1). In the patient group, mean VAS was $6.6 \pm 1.7$ and mean ODI was $33.3 \pm 14.4$.

\section{Endplate BML prevalence}

Twenty-seven subjects ( 26 patients, 1 control) presented with at least one level having endplate BML, and BML presence was significantly associated with pain status (OR 13.0, 95\% CI 2.6-64.2; $p=0.002$ ). The overall prevalence of endplate BML by type was: MC 1 (4.3\%, 26/600 endplates), MC 2 (7.8\%, 49/600 endplates), and MC 3 (0\%, 0/600 endplates). For all types except MC 3, the prevalence was significantly higher in the CLBP group (Table 1). Inter-rater reliability of MC classification was excellent $(\kappa=0.838)$.

\section{Mean BMF - ROI analysis}

In endplates with $\mathrm{MC}$, mean $\mathrm{BMF}$ was altered compared to endplates without MC (Fig. 1a). Specifically, for endplates with fibrovascular BML (MC 1), mean BMF was significantly lower than in endplates without MC (absolute difference $-22.3 \%$; 95\% CI $-24.8,-19.8 ; p<0.001)$. For endplates with fatty BML (MC 2), mean BMF was significantly higher (absolute difference $21.0 \%, 95 \%$ CI 19.1, 22.9; $p<0.001)$. For both MC types, mean BMF in the affected endplate was also significantly different compared to unaffected regions of the same vertebral body (Fig. 1b). For levels without MC, mean BMF was positively associated with age $(p<0.001)$ and higher in males than females $(p=0.012)$.

\section{BMF distributions - vertebral body analysis}

Table 2 shows the values of the six parameters derived from the BMF distributions of the segmented vertebral bodies. Each parameter differed significantly between vertebral bodies with and without MC (mean, standard deviation, coefficient of variation, full width at half maximum) or differed significantly between vertebral bodies with MC 1 versus MC 2 (mean, median, coefficient of variation, skewness). For example, the low BMF values inside MC 1 resulted in left-leaning distributions compared to the high BMF values inside MC 2, which resulted in right-leaning distributions (Fig. 2). Overall, MC presence was associated with BMF 
a

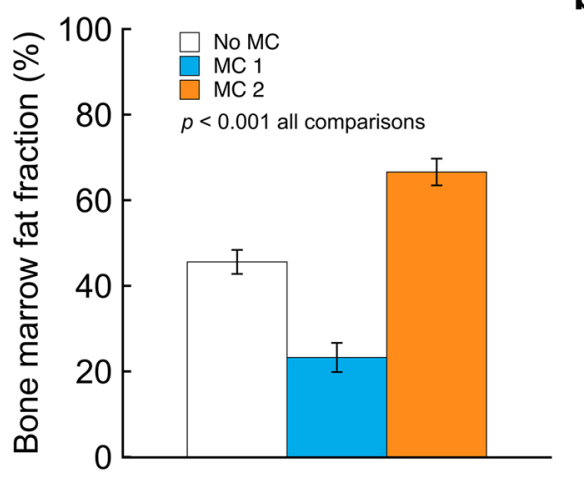

C $\mathrm{F} 55-\mathrm{MC} 1$

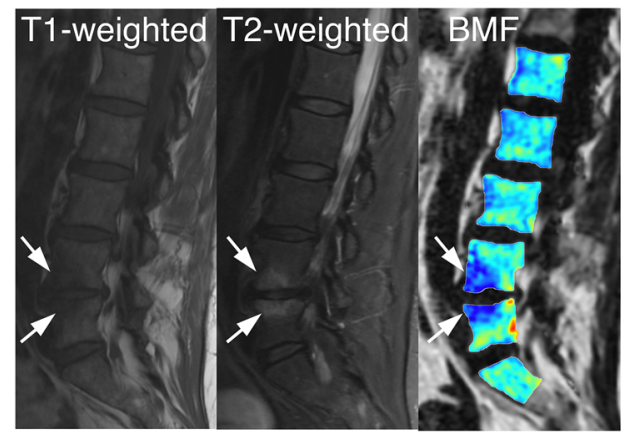

M36 - No MC

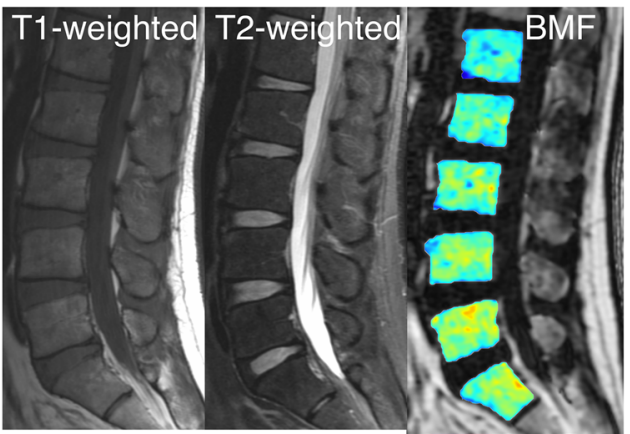

Fig. 1 (a) Comparison of bone marrow fat fraction (mean $\pm 95 \% \mathrm{CI}$ ) between endplates with and without Modic changes (MC). Mean fat fractions were as follows: no MC, $45.6 \%$ (95\% CI: 42.8-48.4; $n=525$ endplates); MC 1, 23.3\% (95\% CI: 19.9-26.7; $n=26$ endplates); MC 2, 66.6\% (95\% CI: 63.5-69.7; $n=49$ endplates). Mixed effects models were used to estimate the least squares mean fat fraction, accounting for multiple measurements per subject; Tukey's HSD tests were used compare the mean fat fraction between different MC types. (b) Pairwise differences in bone marrow fat fraction between the affected

distributions that were more heterogeneous, having shorter and broader peaks.

\section{Associations between BMF measures and clinical outcomes}

For patients with endplate BML, the absolute differences in BMF between affected and unaffected marrow and unaffected regions of endplates with MC. Differences greater than zero indicate higher fat fraction in MC; differences less than zero indicate lower fat fraction in MC. $\mathrm{P}$ values show the result of paired $t$ tests for all endplates with each type of MC. (c) Representative images of patients with and without MC. Arrows point to endplates with $M C$ identified using the $\mathrm{T}_{1}$ and fat-saturated $\mathrm{T}_{2}$ images. The BMF maps are shown with an overlay of the employed segmentation masks

were positively associated with ODI $(p=0.029-0.032$; Fig. 3). These composite BMF measures were not associated with the VAS pain intensity scale $(p=0.49-0.83)$, age $(p=0.06-0.17)$, sex $(p=0.21-0.67)$, Pfirrmann grade $(p=0.10-0.11)$, or MC type $(p=0.19-0.80)$. 
Table 2 Characteristics of bone marrow fat fraction (BMF) distributions derived from segmentations of vertebral bodies with and without Modic changes (MC)

\begin{tabular}{|c|c|c|c|c|}
\hline Characteristic & No MC $(n=296)$ & $\operatorname{MC} 1(n=24)$ & $\operatorname{MC} 2(n=40)$ & $P$ value \\
\hline Mean BMF (\%) & $42.7(40.3,45.1)$ & $40.3(37.4,43.2)^{\mathrm{c}}$ & $46.7(43.9,49.4)^{\mathrm{a}, \mathrm{b}}$ & $<0.001$ \\
\hline Median BMF (\%) & $43.3(40.8,45.8)$ & $41.9(38.9,44.8)$ & $46.7(43.9,49.5)^{\mathrm{a}, \mathrm{b}}$ & $<0.001$ \\
\hline Standard deviation $(\%)$ & $9.2(8.8,9.6)$ & $12.4(11.7,13.1)^{\mathrm{a}}$ & $12.6(12.0,13.2)^{\mathrm{a}}$ & $<0.001$ \\
\hline Coefficient of variation (\%) & $22.3(21.0,23.6)$ & $32.8(29.9,33.7)^{\mathrm{a}}$ & $28.4(26.7,30.1)^{\mathrm{a}, \mathrm{b}}$ & $<0.001$ \\
\hline Skewness & $-0.41(-0.51,-0.29)$ & $-0.39(-0.58,-0.20)$ & $0.06(-0.10,0.23)^{\mathrm{a}, \mathrm{b}}$ & $<0.001$ \\
\hline Full width at half $\max (\%)$ & $15.6(14.4,16.7)$ & $18.1(15.9,20.3)^{\mathrm{c}}$ & $17.9(16.0,19.8)^{\mathrm{c}}$ & 0.002 \\
\hline
\end{tabular}

Data in parentheses are 95\% confidence intervals. Mixed effects models accounting for multiple measurements per subject were used to estimate the least squares mean of each characteristic derived from the segmented BMF maps. Tukey-Kramer post hoc tests were used to test for differences in the least squares mean of each characteristic between groups. $B M F$ bone marrow fat fraction, coefficient of variation $=\mathrm{SD} / \mathrm{mean}$

${ }^{\mathrm{a}} p<0.001$ versus No MC

${ }^{\mathrm{b}} p<0.001$ versus MC1

${ }^{\mathrm{c}} p<0.05$ versus No MC

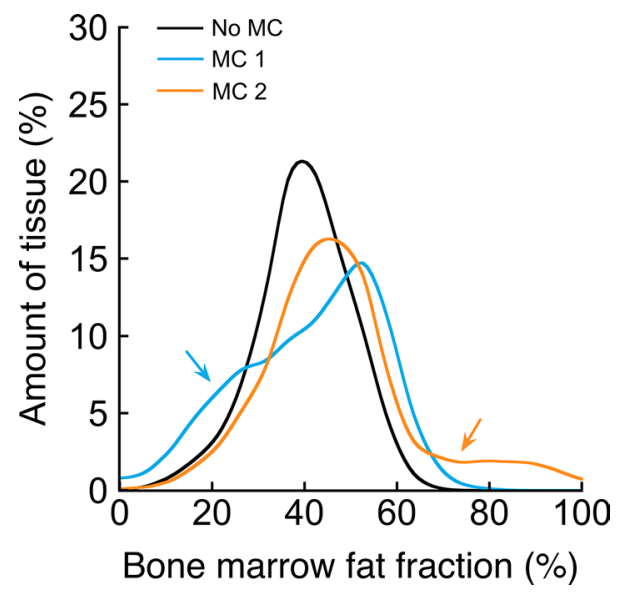

Fig. 2 Histograms showing representative distributions of vertebral BMF values derived from water-fat MRI. Low BMF values inside MC 1 (blue arrow) resulted in right-skewed (left leaning) distributions compared to the high BMF values inside MC 2 (orange arrow), which resulted in left-skewed (right leaning) distributions. In both instances, MC presence within the vertebral body associated with BMF distributions having shorter and wider peaks compared to BMF distributions from vertebral bodies without MC

\section{Repeatability of water-fat MRI}

Test-retest repeatability of the imaging protocol and segmentation procedure indicated low inter-test variability in BMF relative to inter-subject variability (Fig. 4). The overall precision errors in mean BMF values were 3.43\% (L4), 3.33\% (L5), and $4.04 \%$ (S1).

\section{Discussion}

We used IDEAL water-fat separation to measure bone marrow fat for the first time in CLBP patients with endplate BML. The data indicate that BMF was significantly altered in endplate BML: in MC 1, BMF was $22.3 \%$ lower $(p<0.001)$, and in MC 2, BMF was $21.0 \%$ higher $(p<0.001)$ than in unaffected marrow. The nature of those alterations agrees with histopathology reports of fibrovascular and fatty replacement of the bone marrow in endplate BML that appear as MC 1 and MC 2, respectively [6, 18], which support BMF as a quantitative and continuous assessment of marrow composition that reflects the pathophysiology of MC. Our findings also reveal increased heterogeneity in vertebral BMF distributions for levels with endplate BML, which may have value for objectively diagnosing MC presence. Finally, whereas past work explored the clinical significance of endplate BML size [19, 20], our present findings establish the potential importance of BML composition. Specifically, we found the absolute difference in BMF between affected and unaffected marrow varied widely, even between patients with a particular MC type, and larger differences in BMF were associated with more severe disability $(p=0.029-0.032)$. This suggests that these BMF measures may relate to the clinical presentation of CLBP patients with MC. The pathogenesis and clinical relevance of endplate BML is controversial, owing partly to the qualitative and binary assessment methodology [2]. Taken together, our present findings suggest that evaluation of vertebral BMF provides measures of BML composition that can form the basis for quantitative and continuous assessments. This could clarify BML progression and inform treatment development and patient phenotyping.

The sensitivity of the IDEAL method for detecting subtle differences in BMF within and between individuals has 


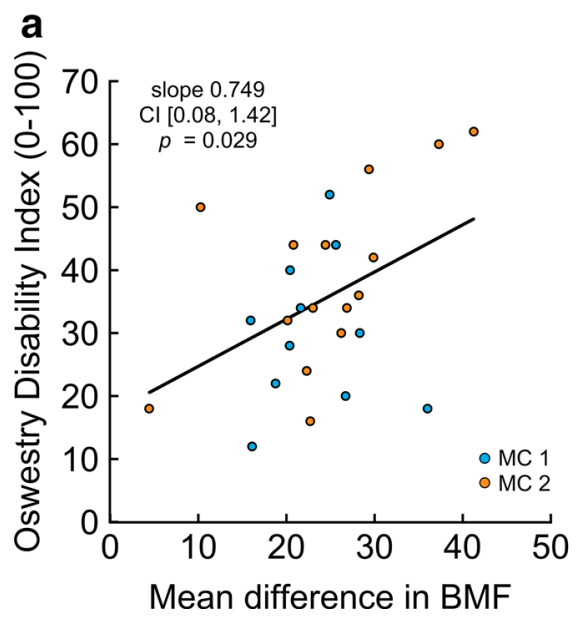

Fig. 3 Scatterplots show associations between composite BMF measures and patient-reported disability ( $n=26$ patients). Composite BMF measures were defined using the absolute differences in bone marrow fat fraction (BMF) between affected and unaffected regions for each level with an endplate BML. Higher composite BMF values indicate greater absolute differences between affected and unaffected marrow in a particular patient. (a) The mean difference in BMF for all levels b

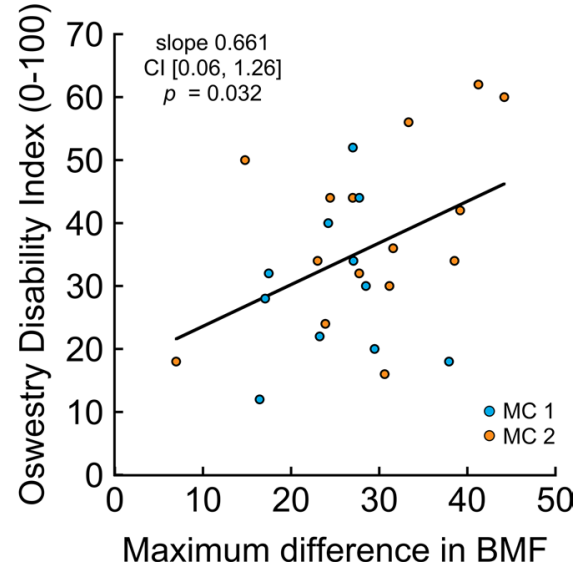

with endplate BML in a particular patient was positively associated with disability. (b) The maximum difference in BMF for all levels with endplate BML in a particular patient was positively associated with disability. Note that for patients with only one level with endplate BML, the mean difference in BMF and the maximum difference in BMF are equivalent
Fig. 4 Test-retest repeatability of water-fat imaging and bone marrow fat fraction (BMF) segmentation for two subjects that were imaged three times after exiting and re-entering the scanner between tests. The curves in each histogram show the distribution of BMF values for each test for the vertebral bodies of (a) L4, (b) L5, and (c) S1, the three levels with the highest incidence of endplate BML seen on MRI. (d) Representative images showing qualitative repeatability of segmented BMF maps for the same subjects
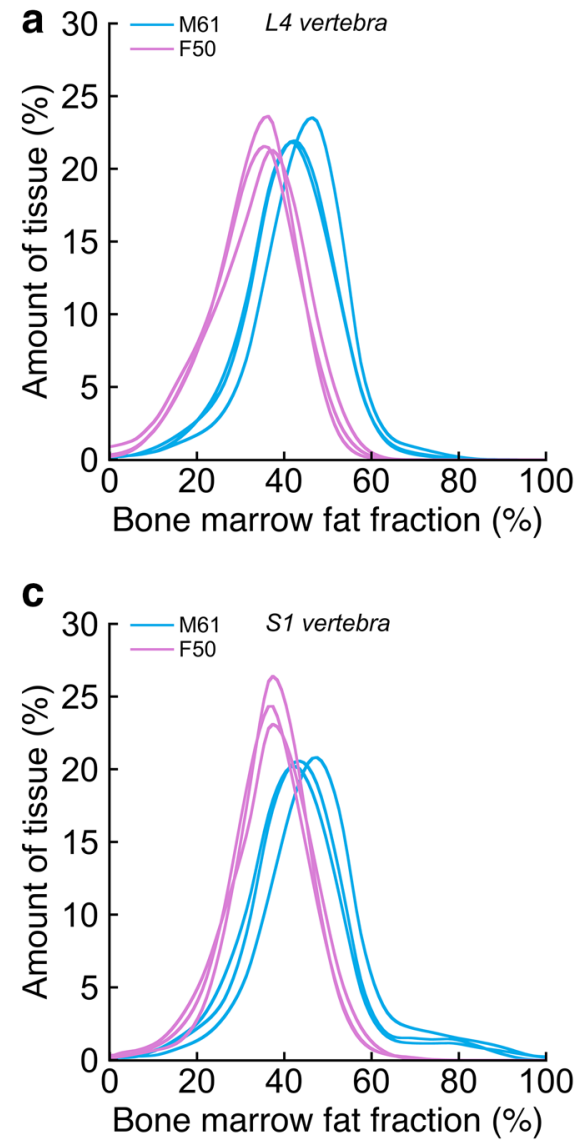

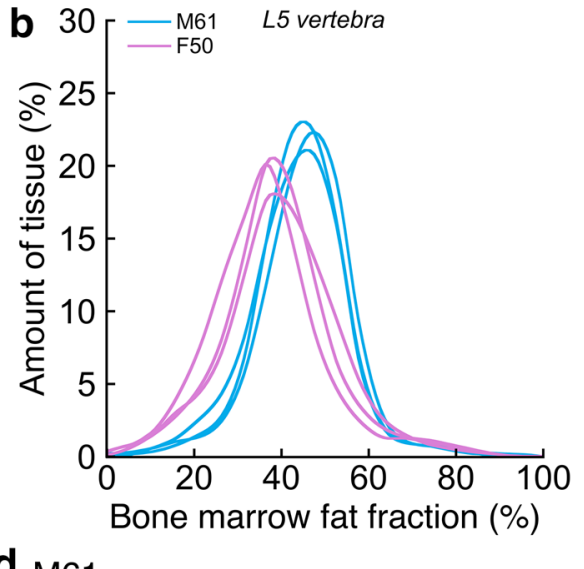

d M61 $_{1}$

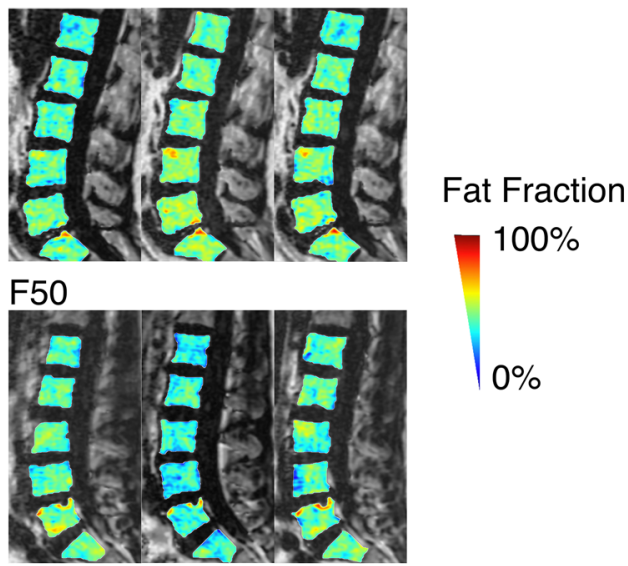


potential clinical implications for quantitative evaluation of endplate BML. The progression of endplate BML in relation to symptoms is unclear [21] and is hindered by the dynamic clinical presentation and by the lack of quantitative tools for measuring the composition of the underlying marrow. For example, previous studies reported that MC 1 and MC 2 are inter-convertible [22, 23] and that approximately $20 \%$ of BML show characteristics of both MC 1 and MC 2 or MC 2 and MC 3 [24]. This confounds binary MC classification, which could partly explain why the size of any one type of MC may not directly correlate with symptoms [20]. Compared to binary classification, continuous quantitative assessments of BMF appear well suited for capturing the dynamic and heterogeneous pathophysiology of endplate BML. For example, we found that heightened BMF values inside MC 2 were often marginated by a narrow band of low BMF values (Fig. 1c), consistent with active conversion. Also, for a particular MC type, the differences in BMF between affected and unaffected marrow spanned a wide range (Fig. 1c; MC 1: $-37.9 \%$ to $-14.3 \%$; MC $2 ;-2.5 \%$ to $44.2 \%$ ), indicating that binary classification belies the heterogeneity in marrow composition. Moreover, the significant associations between those BMF differences and ODI suggest clinical relevance of measuring BML composition. This is further corroborated by results from a histopathology study indicating that the degree of innervation in endplate BML - a theoretical basis for nociceptive pain-may associate with BML composition but not with BML size [18]. Although requiring confirmation in longitudinal studies, these data collectively suggest that BMF assessment may aid in clarifying BML progression in relation to symptoms.

Our framework using water-fat MRI is based on a 6-echo SPGR sequence with IDEAL reconstruction algorithm. Other approaches used for augmenting endplate BML diagnosis include chemical shift imaging (e.g. Dixon), short T1 inversion recovery (STIR), diffusion-weighted imaging (DWI), and ultrashort echo time (UTE) imaging. However, STIR is mainly used to confirm marrow oedema, DWI may differentiate between MC 1 with degenerative vs. infectious aetiologies [25], and UTE imaging can distinguish the bony endplate from cartilage endplate [13]. We are not aware of prior studies that measured marrow composition in MC; nonetheless, mean $\mathrm{BMF}$ in non-MC regions was in the range reported for similarly aged adults $[9,10]$, which supports our findings' validity.

This study has limitations. First, it is a single-site, single-scanner study with a small number of CLBP patients. Although MC prevalence in the CLBP group (26/42 patients, $61.9 \%$ ) was in the range found previously [1], there were only 11 patients with MC 1 and 15 patients with MC 2 . A larger number of MC cases, including MC 3 , are needed to explore the role of BML size and to investigate whether the relationship between the composite BMF measures and clinical outcomes depends on MC type or the severity of disc degeneration. Related, although endplate BML are highly specific predictors of CLBP [1, 2], other factors may contribute to disability and pain sensation independently. Second, this study was cross-sectional, which could partly explain the modest associations with clinical presentation; also, we were unable to determine how changes in BMF relate to changes in clinical characteristics. Nevertheless, our findings motivate BMF assessment in the future longitudinal studies.

In summary, we found that vertebral BMF was significantly altered in CLBP patients with endplate BML and that for a given patient, larger differences in BMF between affected and unaffected marrow were associated with more severe disability. Taken together, these data demonstrate that IDEAL water-fat separation enables measures of marrow composition that can form the basis for quantitative and continuous assessments of endplate BML. Future studies will use this approach to clarify BML progression, inform treatment development, and guide patient selection.

Supplementary Information The online version contains supplementary material available at https://doi.org/10.1007/s00586-021-06738-y.

Acknowledgements The authors thank the following individuals for their help with subject recruitment: Alice Rochette, Kaitlyn Gary, Emel Ece Ozcan, Hemra Cil, and John Callander. This research was supported by the National Institutes of Health (R01AR070198, R01AR063705, P30AR075055) and through the NIH HEAL Initiative under award numbers UH2AR076719 and U19AR076737. The content is solely the responsibility of the authors and does not necessarily represent the official views of the National Institutes of Health or its NIH HEAL Initiative.

Open Access This article is licensed under a Creative Commons Attribution 4.0 International License, which permits use, sharing, adaptation, distribution and reproduction in any medium or format, as long as you give appropriate credit to the original author(s) and the source, provide a link to the Creative Commons licence, and indicate if changes were made. The images or other third party material in this article are included in the article's Creative Commons licence, unless indicated otherwise in a credit line to the material. If material is not included in the article's Creative Commons licence and your intended use is not permitted by statutory regulation or exceeds the permitted use, you will need to obtain permission directly from the copyright holder. To view a copy of this licence, visit http://creativecommons.org/licenses/by/4.0/.

\section{References}

1. Jensen TS, Karppinen J, Sorensen JS, Niinimaki J, Leboeuf-Yde C (2008) Vertebral endplate signal changes (Modic change): a systematic literature review of prevalence and association with non-specific low back pain. Eur Spine J 17:1407-1422. https:// doi.org/10.1007/s00586-008-0770-2

2. Fields AJ, Battie MC, Herzog RJ, Jarvik JG, Krug R, Link TM, Lotz JC, O'Neill CW, Sharma A, Group IDSP (2019) Measuring and reporting of vertebral endplate bone marrow lesions as seen on MRI (Modic changes): recommendations from the ISSLS 
Degenerative Spinal Phenotypes Group. Eur Spine J 28:22662274. https://doi.org/10.1007/s00586-019-06119-6

3. Chen Y, Yang H, Zhang L, Wang Y, Zou J (2019) Analyzing the influence of Modic changes on patients with lower back pain undergoing conservative treatment. Pain Res Manag 2019:8185316. https://doi.org/10.1155/2019/8185316

4. Jensen OK, Nielsen CV, Sorensen JS, Stengaard-Pedersen K (2014) Type 1 Modic changes was a significant risk factor for 1-year outcome in sick-listed low back pain patients: a nested cohort study using magnetic resonance imaging of the lumbar spine. Spine J 14:2568-2581. https://doi.org/10.1016/j.spine e.2014.02.018

5. Khalil J, Smuck M, Koreckij T, Keel J, Beall D, Goodman B, Kalapos P, Nguyen D, Garfin S, Investigators IT (2019) A prospective, randomized, multi-center study of intraosseousbasivertebral nerve ablation for the treatment of chronic low back pain. Spine J. https://doi.org/10.1016/j.spinee.2019.05.598

6. Modic MT, Steinberg PM, Ross JS, Masaryk TJ, Carter JR (1988) Degenerative disk disease: assessment of changes in vertebral body marrow with MR imaging. Radiology 166:193-199

7. Karampinos DC, Melkus G, Baum T, Bauer JS, Rummeny EJ, Krug R (2014) Bone marrow fat quantification in the presence of trabecular bone: initial comparison between water-fat imaging and single-voxel MRS. MagnReson Med 71:1158-1165. https:// doi.org/10.1002/mrm. 24775

8. Karampinos DC, Ruschke S, Dieckmeyer M, Diefenbach M, Franz D, Gersing AS, Krug R, Baum T (2018) Quantitative MRI and spectroscopy of bone marrow. J MagnReson Imaging 47:332-353. https://doi.org/10.1002/jmri.25769

9. Arentsen L, Yagi M, Takahashi Y, Bolan PJ, White M, Yee D, Hui S (2015) Validation of marrow fat assessment using noninvasive imaging with histologic examination of human bone samples. Bone 72:118-122. https://doi.org/10.1016/j.bone.2014.11.002

10. MacEwan IJ, Glembotski NE, D'Lima D, Bae W, Masuda K, Rashidi HH, Mell LK, Bydder M (2014) Proton density water fraction as a biomarker of bone marrow cellularity: validation in ex vivo spine specimens. MagnReson Imaging 32:1097-1101. https://doi.org/10.1016/j.mri.2014.03.005

11. Baum T, Yap SP, Dieckmeyer M, Ruschke S, Eggers H, Kooijman H, Rummeny EJ, Bauer JS, Karampinos DC (2015) Assessment of whole spine vertebral bone marrow fat using chemical shiftencoding based water-fat MRI. J MagnReson Imaging 42:10181023. https://doi.org/10.1002/jmri.24854

12. Krug R, Joseph GB, Han M, Fields A, Cheung J, Mundada M, Bailey J, Rochette A, Ballatori A, McCulloch CE, McCormick Z, O'Neill C, Link TM, Lotz J (2019) Associations between vertebral body fat fraction and intervertebral disc biochemical composition as assessed by quantitative MRI. J MagnReson Imaging. https:// doi.org/10.1002/jmri.26675

13. Bailey JF, Fields AJ, Ballatori A, Cohen D, Jain D, Coughlin D, O'Neill C, McCormick Z, Han M, Krug R, Demir-Deviren S, Lotz JC (2019) The relationship between endplate pathology and patient-reported symptoms for chronic low back pain depends on lumbar paraspinal muscle quality. Spine (Phila Pa 1976). doi: https://doi.org/10.1097/BRS.0000000000003035

14. Gee CS, Nguyen JT, Marquez CJ, Heunis J, Lai A, Wyatt C, Han M, Kazakia G, Burghardt AJ, Karampinos DC, Carballido-Gamio J, Krug R (2015) Validation of bone marrow fat quantification in the presence of trabecular bone using MRI. J MagnReson Imaging 42:539-544. https://doi.org/10.1002/jmri.24795

15. Reeder SB, Pineda AR, Wen Z, Shimakawa A, Yu H, Brittain JH, Gold GE, Beaulieu CH, Pelc NJ (2005) Iterative decomposition of water and fat with echo asymmetry and least-squares estimation (IDEAL): application with fast spin-echo imaging. MagnReson Med 54:636-644. https://doi.org/10.1002/mrm.20624

16. Corbeil RR, Searle SR (1976) Restricted maximum likelihood (Reml) estimation of variance components in mixed model. Technometrics 18:31-38. https://doi.org/10.2307/1267913

17. Gluer CC, Blake G, Lu Y, Blunt BA, Jergas M, Genant HK (1995) Accurate assessment of precision errors: how to measure the reproducibility of bone densitometry techniques. OsteoporosInt 5:262-270

18. Fields AJ, Liebenberg EC, Lotz JC (2014) Innervation of pathologies in the lumbar vertebral end plate and intervertebral disc. Spine J 14:513-521. https://doi.org/10.1016/j.spinee.2013.06.075

19. Weishaupt D, Zanetti M, Hodler J, Min K, Fuchs B, Pfirrmann CW, Boos N (2001) Painful lumbar disk derangement: relevance of endplate abnormalities at MR imaging. Radiology 218:420-427

20. Kaapa E, Luoma K, Pitkaniemi J, Kerttula L, Gronblad M (2012) Correlation of size and type of modic types 1 and 2 lesions with clinical symptoms: a descriptive study in a subgroup of patients with chronic low back pain on the basis of a university hospital patient sample. Spine (Phila Pa 1976) 37:134-139. doi: https:// doi.org/10.1097/BRS.0b013e3182188a90

21. Luoma K, Vehmas T, Kerttula L, Gronblad M, Rinne E (2016) Chronic low back pain in relation to Modic changes, bony endplate lesions, and disc degeneration in a prospective MRI study. Eur Spine J 25:2873-2881. https://doi.org/10.1007/s0058 6-016-4715-x

22. Jensen TS, Bendix T, Sorensen JS, Manniche C, Korsholm L, Kjaer P (2009) Characteristics and natural course of vertebral endplate signal (Modic) changes in the Danish general population. BMC MusculoskeletDisord 10:81. https://doi. org/10.1186/1471-2474-10-81

23. Schmeel FC, Vomweg T, Traber F, Gerhards A, Enkirch SJ, Faron A, Sprinkart AM, Schmeel LC, Luetkens JA, Thomas D, Kukuk GM (2019) Proton density fat fraction MRI of vertebral bone marrow: Accuracy, repeatability, and reproducibility among readers, field strengths, and imaging platforms. J MagnReson Imaging 50:1762-1772. https://doi.org/10.1002/jmri.26748

24. Wang Y, Videman T, Battie MC (2012) Lumbar vertebral endplate lesions: prevalence, classification, and association with age. Spine (Phila Pa 1976) 37:1432-1439. doi: https://doi.org/10.1097/ BRS.0b013e31824dd20a

25. Patel KB, Poplawski MM, Pawha PS, Naidich TP, Tanenbaum LN (2014) Diffusion-weighted MRI "claw sign" improves differentiation of infectious from degenerative modic type 1 signal changes of the spine. AJNR Am J Neuroradiol 35:1647-1652. https://doi. org/10.3174/ajnr.A3948

Publisher's Note Springer Nature remains neutral with regard to jurisdictional claims in published maps and institutional affiliations. 